\title{
Pneumocystis jirovecii Pneumonia-Associated Acute Respiratory Distress Syndrome Complicated by Pneumomediastinum and Pneumopericardium in a Non-Human Immunodeficiency Virus-Infected Patient
}

\author{
Jose Orsini ${ }^{\mathrm{a}}$, , Hannah Gawlak ${ }^{\mathrm{a}}$, Vladimir Sabayev ${ }^{\mathrm{a}}$, Kumar Shaha, Leah Washburn ${ }^{\mathrm{a}}$, \\ Keira McCarthy ${ }^{\mathrm{a}}$, Anthony Courey ${ }^{\mathrm{a}}$, Erin Mouyeos ${ }^{\mathrm{a}}$, Siblea Pangallo ${ }^{\mathrm{a}}$
}

\begin{abstract}
Pneumocystis jirovecii pneumonia is widely known as a life-threatening opportunistic infection in patients with acquired immunodeficiency syndrome (AIDS). However, with the widespread use of highly active antiretroviral therapy (HAART) and effective anti-Pneumocystis antimicrobial prophylaxis, this entity has declined substantially in patients with human immunodeficiency virus (HIV) infection. Interestingly, the incidence of Pneumocystis jirovecii pneumonia has been increasing among patients without HIV infection, mainly as a consequence of the expanding use of chemotherapy and other immunosuppressive agents. Nevertheless, Pneumocystis jirovecii pneumonia remains an important cause of HIV- and non-HIV-related catastrophic complications. Pneumomediastinum and pneumopericardium are extremely uncommon events in patients with Pneumocystis jirovecii pneumonia. In this report, we described a unique case of Pneumocystis jirovecii pneumonia-associated acute respiratory distress syndrome (ARDS), complicated by pneumomediastinum and pneumopericardium in a non-HIV infected patient.
\end{abstract}

Keywords: Pneumocystis jirovecii; ARDS; Pneumomediastinum; Pneumopericardium; HIV-negative

\section{Introduction}

Despite advances in modern intensive care medicine, with standardized strategies against acute respiratory distress syn-

Manuscript submitted December 20, 2019, accepted January 21, 2020

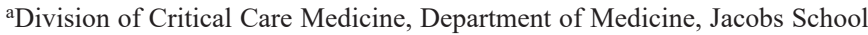
of Medicine and Biomedical Sciences at University of Buffalo, Mercy Hospital of Buffalo, 565 Abbott Road, Buffalo, NY 14220, USA

${ }^{b}$ Corresponding Author: Jose Orsini, Division of Critical Care Medicine, Department of Medicine, Jacobs School of Medicine and Biomedical Sciences at University of Buffalo, Mercy Hospital of Buffalo, 565 Abbott Road, Buffalo, NY 14220, USA. Email: joseorsini@yahoo.com

doi: https://doi.org/10.14740/jocmr4074 drome (ARDS), Pneumocystis jirovecii pneumonia accounts for substantial morbidity and mortality among non-human immunodeficiency virus (HIV)-infected patients. The incidence of Pneumocystis jirovecii pneumonia has been increasing in patients without HIV infection, with reported higher mortality rates between $30-60 \%$ as compared to HIV-infected patients [1-3]. Although the reasons for these differences in mortality rates are not fully understood, it has been hypothesized that the higher neutrophil counts in bronchoalveolar lavage (BAL) fluid observed in HIV-negative patients with Pneumocystis jirovecii pneumonia may be an important factor [4]. HIV-negative patients have 10-fold higher incidence of endotracheal intubation and mechanical ventilation, suggesting that non-HIV infected patients have a more intense inflammatory response. Risk factors for increased mortality among non-HIV-infected patients with Pneumocystis jirovecii pneumonia requiring mechanical ventilation includes: the need for positive end-expiratory pressure (PEEP) during the first 3 days of therapy, the development of pneumothorax during mechanical ventilation, elevated alveolar-arterial oxygen gradient, concurrent infection with cytomegalovirus (CMV) and Aspergillus spp, lymphopenia, and pre-existing lung disease [3, 5-7]. Pneumocystis jirovecii pneumonia is a rare cause of pneumomediastinum that has been previously described in both HIV-positive [8,9] and HIVnegative patients [10]. However, pneumopericardium complicating Pneumocystis jirovecii pneumonia has not been reported among HIV-negative patients. Park et al reported a case of spontaneous pneumomediastinum, pneumopericardium, and pneumothorax in a patient with acquired immunodeficiency syndrome (AIDS) and Pneumocystis jirovecii pneumonia [11]. Hence, we report the first case of a combined pneumomediastinum and pneumopericardium in an HIV-negative patient with Pneumocystis jirovecii pneumonia.

\section{Case Report}

A 68-year-old male was transferred from another institution because of progressively worsening tachypnea for 1 month, with increased fraction of inspired oxygen $\left(\mathrm{FIO}_{2}\right)$ requirements that led to endotracheal intubation and initiation of mechanical 


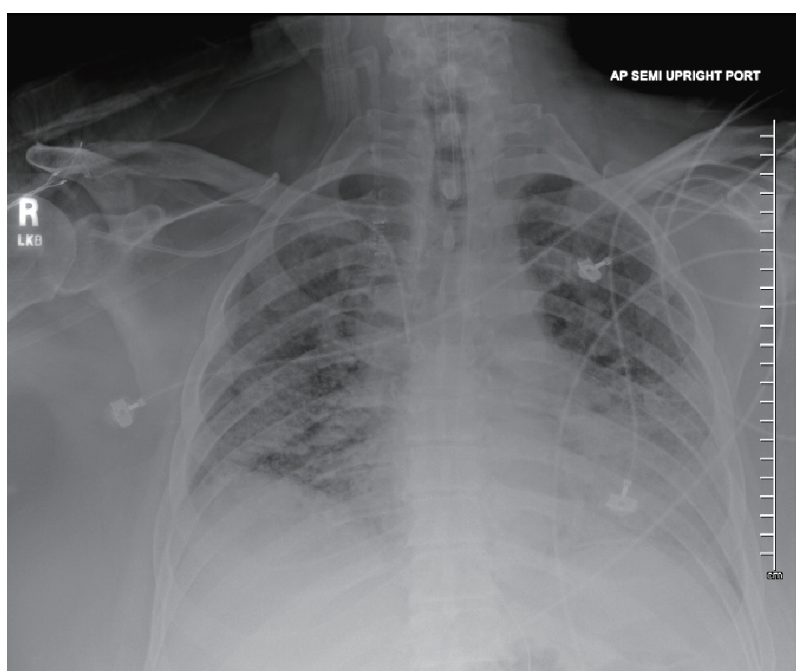

Figure 1. Chest X-ray showing bilateral increased interstitial markings.

ventilation. His past medical history was significant for morbid obesity (body mass index of $47 \mathrm{~kg} / \mathrm{m}^{2}$ ), systemic arterial hypertension, diabetes mellitus, and a chronic lung disease of unclear etiology. Heavy smoking history for several years was reported by patient's relatives. His outpatient medications regimen included ramipril, amlodipine, metformin and metoprolol. There was no evidence of recent or remote exposure to corticosteroids therapy or any other immunosuppressive agents. Vital signs on admission were as follows: blood pressure of $86 / 52 \mathrm{~mm} \mathrm{Hg}$ (mean arterial pressure of 63), a regular heart rate of 104 beats/ min, a respiratory rate of 24 breaths/min, and a temperature of $37^{\circ} \mathrm{C}$. Physical examination was remarkable for bilateral rales and wheezing on lung auscultation. Notable laboratory findings included a white blood cell (WBC) count of $16.4 \times 10^{3} / \mathrm{mm}^{3}$ (4 - 11), an alanine aminotransferase (ALT) level of $119 \mathrm{U} / \mathrm{L}$ ( 7 - 52), a lactate dehydrogenase (LDH) level of $488 \mathrm{U} / \mathrm{L}$ (105 - 210), and a blood urea nitrogen (BUN) level of $36 \mathrm{mg} / \mathrm{DL}$ (8 - 27). Creatinine level, cardiac enzymes and coagulation profile were within normal limits, and HIV test was negative. Arterial blood gas $(\mathrm{ABG})$ while on mechanical ventilation and receiving an $\mathrm{FIO}_{2}$ of $60 \%$ and a PEEP of $10 \mathrm{~cm} \mathrm{H}_{2} \mathrm{O}$ showed a $\mathrm{pH}$ of 7.24 (7.35 - 7.45), a pCO $\mathrm{pl}_{2}$ level of $69 \mathrm{~mm} \mathrm{Hg} \mathrm{(35} \mathrm{-} \mathrm{45),} \mathrm{and} \mathrm{a}$ $\mathrm{PaO}_{2}$ level of $67 \mathrm{~mm} \mathrm{Hg}(75-100)$. Initial chest X-ray (CXR) showed bilateral increased interstitial markings with a possible left retrocardiac consolidation (Fig. 1). Chest computed tomography $(\mathrm{CT})$ disclosed bilateral ground-glass opacities, without evidence of pulmonary embolism. Venous Doppler ultrasound of lower extremities demonstrated no evidence of deep venous thrombosis. Electrocardiogram (ECG) revealed sinus tachycardia without ST-segment or T-wave abnormalities. Transthoracic echocardiogram (TTE) disclosed a normal left ventricular ejection fraction, with a normal right ventricular systolic function, and no evidence of pulmonary hypertension.

Patient was admitted to the intensive care unit (ICU), and empiric intravenous antimicrobial therapy composed by vancomycin (15 mg/kg Q12h), cefepime (2 g Q8h), and azithromycin (500 mg daily), along with vasopressors (norepinephrine continuous infusion) was initiated for presumptive septic shock

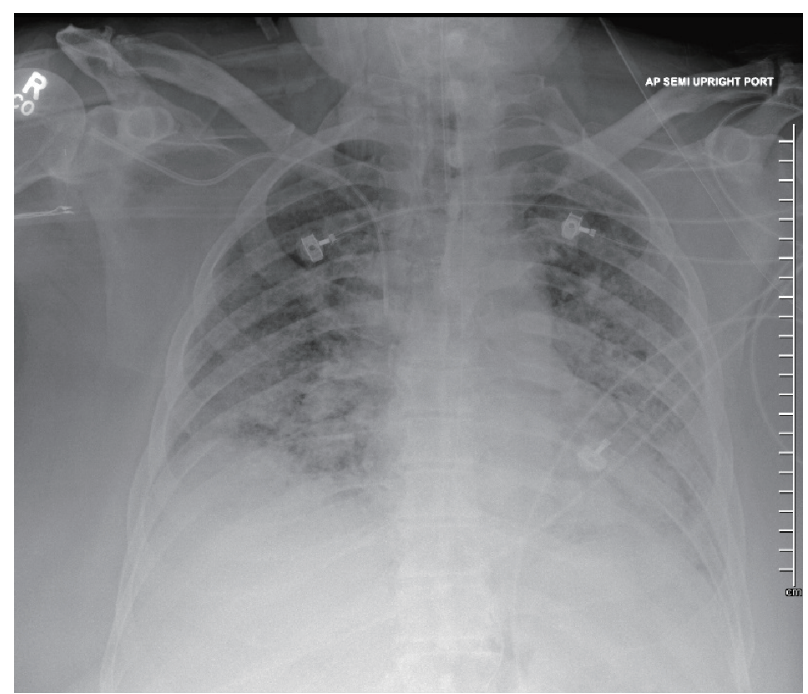

Figure 2. Chest $\mathrm{X}$-ray demonstrating worsening of bilateral opacities.

caused by a severe community-acquired pneumonia (CAP). Blood and respiratory cultures as well as Legionella and Streptococcus pneumoniae urinary antigens and serum cryptococcal antigen were negative; and all antimicrobials were discontinued after completion of 7 days of empiric therapy. Procalcitonin level was $0.25 \mathrm{ng} / \mathrm{mL}(0.00-0.10)$. The (1-3)- $\beta$-D-glucan assay (Fungitell ${ }^{\circ}$, Quest Diagnostics) showed the result was $32 \mathrm{pg} / \mathrm{mL}(<60)$. ICU clinical course was complicated by persistently increased $\mathrm{FIO}_{2} / \mathrm{PEEP}$ requirements $(100 \%$ and $18 \mathrm{~cm} \mathrm{H}_{2} \mathrm{O}$, respectively), ARDS with markedly decreased $\mathrm{PaO}_{2} / \mathrm{FIO}_{2}$ ratio despite low tidal volume strategy as recommended by ARDS network, worsening bilateral infiltrates on CXR (Fig. 2), increased vasopressors requirements, and acute kidney injury. Optimal sedation and analgesia were achieved with a combination of fentanyl and propofol continuous infusion, and an infusion of neuromuscular blocking agent (cisatracurium) was initiated to avoid ventilatory dyssynchrony. Epoprostenol was added as a salvage therapy for refractory hypoxemia. Extracorporeal membrane oxygenation (ECMO) therapy was deemed inappropriate given patient's body habitus. In spite of the needs for escalating PEEP levels, our patient's plateau pressures remained $<35 \mathrm{~cm} \mathrm{H}_{2} \mathrm{O}$. On day 6 of ICU admission, a BAL result performed on admission showed a positive deoxyribonucleic acid (DNA) by polymerase chain reaction (PCR) for Pneumocystis jirovecii. CMV antigenemia by PCR was negative. All serum immunoglobulin levels were within normal limits. Complement component 3 (C3), C4 and CH50 levels were diminished. Repeated HIV test was non-reactive. T-lymphocytes cell count (CD4) was 97 cells/ $\mu \mathrm{L}(490$ $-1,740$ ), with an absolute lymphocytes cell count of $377 \times 10^{3} /$ $\mu \mathrm{L}(850$ - 3,900). Intravenous antimicrobial therapy with trimethoprim-sulfamethoxazole (Bactrim ${ }^{\circledR} ; 15 \mathrm{mg} / \mathrm{kg}$ adjusted to creatinine clearance) along with steroids (methylprednisone 40 mg Q12h) was initiated. Repeated chest CT disclosed diffuse ground-glass opacities, severe pneumomediastinum with pneumopericardium, and bilateral pneumothorax (Fig. 3). Patient's clinical condition continued to worsen despite seemingly appropriate antimicrobial therapy, and on day 14 of ICU 


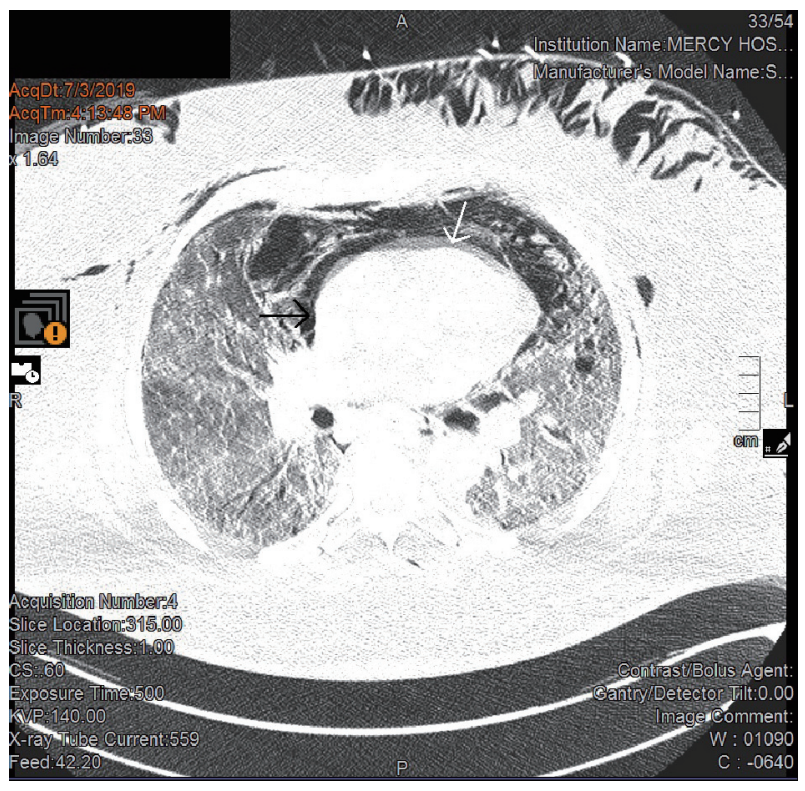

Figure 3. Chest CT showing extensive pneumomediastinum and pneumopericardium. CT: computed tomography.

admission, patient's relatives decided to withdraw all invasive forms of artificial life support. Patient expired few hours later.

\section{Discussion}

Pneumomediastinum and pneumopericardium are uncommon complications in patients with Pneumocystis jirovecii pneumonia requiring mechanical ventilation. Pneumopericardium is usually a consequence of invasive procedures such as mechanical ventilation and placement of central venous catheters [12], while pneumomediastinum frequently developed from increased intrathoracic pressures as a result of asthma or barotraumas, esophageal rupture, cavitating pneumonia, or invasive procedures [13]. The pathophysiology of both pneumomediastinum and pneumopericardium is related to the pressure gradient between the alveoli and lung parenchyma, since increased pressures would lead to alveolar rupture resulting in air leak into interstitial space and mediastinum. The air leak presumably originated from the pneumatoceles formed as a result of proteases released by activated macrophages and/or overdistention resulting from localized inflammation caused by Pneumocystis jirovecii. The free air located in the mediastinum may progress to leak into the pericardial wall and pericardium [8, 14, 15]. Pneumomediastinum and pneumopericardium are both self-limited clinical entities, but physiologic features of cardiac tamponade may require pericardiocentesis with or without chest tube thoracostomy.

Although there have been reported cases of pneumomediastinum and pneumopericardium in patients with HIV infection $[11,16-20]$, there are no documented cases of pneumopericardium among non-HIV infected patients. Based on a MEDLINE search and using the words "pneumomediastinum", "HIV-negative", "non-HIV", "pneumopericardium", and "Pneumocystis jirovecii", the authors identified a total of 51 cases of pneumomediastinum associated with Pneumocystis jirovecii pneumonia demonstrated by chest CT [10, 21-26], but no cases of pneumopericardium were distinguished during that search. The incidence of pneumomediastinum among HIV-negative patients with Pneumocystis jirovecii pneumonia has been reported to range between $11 \%$ and $24 \%[21,24]$. Chest CT findings in Pneumocystis jirovecii pneumonia are similar in both HIVpositive and non-HIV infected patients, ranging from diffuse ground-glass opacities, centrilobular nodules, air bronchograms, consolidations, cysts and pneumatoceles, pleural effusions, hilar and mediastinal lymphadenopathies, bronchiectasis, pneumothorax, and pneumomediastinum [21, 23, 24]. To the best of our knowledge, this is the first report of a combined pneumomediastinum and pneumopericardium in a non-HIV infected patient with Pneumocystis jirovecii pneumonia.

It is difficult to establish how our patient acquired Pneumocystis jirovecii pneumonia, given the fact that the only known immunosuppression was diabetes mellitus. We hypothesized that the chronic lung disease mention as a part of patient's medical history may had been a form of an undiagnosed interstitial lung disease. Furthermore, we suggest that our patient might have been previously colonized with Pneumocystis jirovecii, since the prevalence of carriage and colonization among patients with interstitial lung diseases have been reported to range between $22 \%$ and $63 \%[27,28]$. We also conjecture that, given the history of chronic smoking, our patient probably had chronic obstructive pulmonary disease (COPD). Colonization rates have been reported to be around 30\% among HIV-negative patients with COPD [29]. It has been suggested that smoking may be a risk factor for Pneumocystis jirovecii colonization, as a result of impaired mucociliary clearance and decreased surfactant [27]. In patients with COPD, colonization with Pneumocystis jirovecii is associated with an increased systemic inflammatory response that includes higher peripheral lymphocyte counts and elevated levels of interleukin (IL)6 , IL-8, and tumor necrosis factor (TNF)- $\alpha$. We also postulated that our patient may have had an underlying, non-diagnosed lymphoproliferative or autoimmune disorder, which may have allowed a possible reactivation of Pneumocystis jirovecii. This hypothesis would be sustained by the fact that serum $\mathrm{C} 3, \mathrm{C} 4$, and $\mathrm{CH} 50$ levels were diminished.

The etiology of pneumomediastinum and pneumopericardium in our patient could be explained by two mechanisms: 1) increased intra-alveolar pressure as a result of mechanical ventilation with increased PEEP levels (was as high as $18 \mathrm{~cm}$ $\mathrm{H}_{2} \mathrm{O}$ ), which may lead to rupture of alveoli and air leaking into the mediastinal and pericardial cavities; 2) severe inflammation in the lung parenchyma caused by Pneumocystis jirovecii, which resulted in rupture of necrotic lung tissue caused by proteases released from activated macrophages and/or alveolar overdistention secondary to bronchioalveolar inflammation. It is obvious that our patient had predisposing risk factors for barotrauma: probable COPD, possible interstitial lung disease, Pneumocystis jirovecii, and ARDS. Interestingly, in a large study, Weng et al reported no statistically significant association between elevated levels of PEEP and plateau pressures and the development of pneumomediastinum among non-HIV infected patients receiving mechanical ventilation as a part of 
the therapy for Pneumocystis jirovecii pneumonia [21]. The needs for escalating levels of PEEP in our patient likely represented an ongoing disease process despite appropriate therapy.

Technically, the flexible bronchoscopy performed on day 1 of ICU admission could have possibly caused the observed pneumomediastinum and pneumopericardium in this case, although there are several points arguing against this possible hypothesis. First, the bronchoscopy was performed for diagnostic purposes (BAL) and no tissue biopsy was obtained, so the possibility of iatrogenic pneumomediastinum and pneumopericardium is extremely low. Second, subsequent daily CXRs did not showed any evidence of pneumomediastinum or pneumopericardium, as they were both discovered on a follow-up chest CT on day 7 of ICU admission. Third, a TTE performed on day 3 of ICU admission did not demonstrate any pericardial abnormalities.

Curiously, serum (1-3)- $\beta$-D-glucan level was within normal limits. (1-3)- $\beta$-D-glucan is a polysaccharide that constitutes the major cell wall component of Pneumocystis jirovecii, with reported sensitivity rates ranging from $90 \%$ to $100 \%$ for the detection of Pneumocystis pneumonia. Studies have suggested that $\beta$-glucan levels in serum may correlate to the load of Pneumocystis in respiratory samples, and those levels may be lower in patients colonized with Pneumocystis jirovecii [30, $31]$. Therefore, (1-3)- $\beta$-D-glucan assay sensitivity may be insufficient to rule out Pneumocystis jirovecii pneumonia [32]. It might well be in our case that the Pneumocystis jirovecii infection was in early stage at the time the assay was performed, and that the fungal load was too small to be detected.

The patient described in this report also developed bilateral pneumothorax, a rare condition reported in $2-19 \%$ of HIV-negative patients with Pneumocystis jirovecii pneumonia requiring mechanical ventilation $[33,34]$. The occurrence of pneumothorax in this case is likely related to rupture of subpleural cavities resulting from necrotic lung tissue or emphysematous blebs, as well as gas exchange abnormalities resulted from hypoxemia and the neutrophilic inflammation of the lung parenchyma caused by Pneumocystis jirovecii. Patients without HIV infection seems to have higher incidence of pneumothorax and increased mortality as compared to HIV-infected patients, with fatality rates ranging from $38 \%$ to $100 \%$ in patients with Pneumocystis jirovecii pneumonia requiring mechanical ventilation $[3-5,35,36]$.

In conclusion, this case is unique because it highlights peculiar findings of an uncommon disease: Pneumocystis jirovecii pneumonia in an HIV-negative patient without known immunosuppression, with a normal (1-3)- $\beta$-D-glucan assay result, and combined pneumomediastinum, bilateral pneumothorax, and pneumopericardium. Although pneumomediastinum and pneumopericardium have been infrequently reported in the literature, clinicians should be aware of these potentially catastrophic complications when treating Pneumocystis jirovecii pneumonia in non-HIV infected patients requiring mechanical ventilation.

\section{Acknowledgments}

None to declare.

\section{Financial Disclosure}

This research did not receive any specific grant from any funding agency in the public, commercial or not-for-profit sector.

\section{Conflict of Interest}

None to declare.

\section{Informed Consent}

Not applicable.

\section{Author Contributions}

All authors contributed equally in both medical-clinical management during patient's ICU admission and data searching, literature review, and preparation of this manuscript.

\section{Data Availability}

The authors declare that data supporting the findings of this study are available within the article.

\section{References}

1. Catherinot E, Lanternier F, Bougnoux ME, Lecuit M, Couderc LJ, Lortholary O. Pneumocystis jirovecii Pneumonia. Infect Dis Clin North Am. 2010;24(1):107-138.

2. Thomas CF, Jr., Limper AH. Pneumocystis pneumonia. N Engl J Med. 2004;350(24):2487-2498.

3. Liu CJ, Lee TF, Ruan SY, Yu CJ, Chien JY, Hsueh PR. Clinical characteristics, treatment outcomes, and prognostic factors of Pneumocystis pneumonia in non-HIVinfected patients. Infect Drug Resist. 2019;12:1457-1467.

4. Limper AH, Offord KP, Smith TF, Martin WJ, 2nd. Pneumocystis carinii pneumonia. Differences in lung parasite number and inflammation in patients with and without AIDS. Am Rev Respir Dis. 1989;140(5):1204-1209.

5. Boonsarngsuk V, Sirilak S, Kiatboonsri S. Acute respiratory failure due to Pneumocystis pneumonia: outcome and prognostic factors. Int J Infect Dis. 2009;13(1):59-66.

6. Bedos JP, Dumoulin JL, Gachot B, Veber B, Wolff M, Regnier B, Chevret S. Pneumocystis carinii pneumonia requiring intensive care management: survival and prognostic study in 110 patients with human immunodeficiency virus. Crit Care Med. 1999;27(6):1109-1115.

7. Kim SJ, Lee J, Cho YJ, Park YS, Lee CH, Yoon HI, Lee SM, et al. Prognostic factors of Pneumocystis jirovecii pneumonia in patients without HIV infection. J Infect. 2014;69(1):88-95.

8. Moss S, Carey PB, Hind CR. Pneumocystis carinii pneumonia presenting with pneumomediastinum in an HIV- 
positive patient. Postgrad Med J. 1995;71(832):96-97.

9. Cho JY, Kim DM, Kwon YE, Yoon SH, Lee SI. Newly formed cystic lesions for the development of pneumomediastinum in Pneumocystis jirovecii pneumonia. BMC Infect Dis. 2009;9:171.

10. She WH, Chok KSH, Li IWS, Ma KW, Sin SL, Dai WC, Fung JYY, et al. Pneumocystis jirovecii-related spontaneous pneumothorax, pneumomediastinum and subcutaneous emphysema in a liver transplant recipient: a case report. BMC Infect Dis. 2019;19(1):66.

11. Park YK, Jung HC, Kim SY, Kim MY, Jo K, Kim SY, Kang B, et al. Spontaneous pneumomediastinum, pneumopericardium, and pneumothorax with respiratory failure in a patient with AIDS and pneumocystis jirovecii pneumonia. Infect Chemother. 2014;46(3):204-208.

12. Goodman PC. Radiographic findings in patients with acute respiratory distress syndrome. Clin Chest Med. 2000;21(3):419-433, vii.

13. Marco CA, Rothman RE. HIV infection and complications in emergency medicine. Emerg Med Clin North Am. 2008;26(2):367-387, viii-ix.

14. Abraham GE, 3rd, Sumrall BH, Bowling MR. The air apparent: a rare complication during flexible bronchoscopy. Am J Med Sci. 2011;341(3):243-245.

15. Kaneki T, Kubo K, Kawashima A, Koizumi T, Sekiguchi M, Sone S. Spontaneous pneumomediastinum in 33 patients: yield of chest computed tomography for the diagnosis of the mild type. Respiration. 2000;67(4):408-411.

16. Hosoda T, Sakamoto M. Pneumomediastinum Associated with HIV-positive Pneumocystis jirovecii Pneumonia. Intern Med. 2019;58(24):3607-3608.

17. Kaji Y, Ohara G, Kagohashi K, Satoh H. Pneumomediastinum in a patient with pneumocystis jirovecii pneumonia. Intern Med. 2012;51(16):2251.

18. Chopra V, Garg N, Mrigpuri P. Spontaneous pneumopericardium an unusual complication in a patient of HIV and pulmonary tuberculosis. Lung India. 2013;30(2):148150 .

19. Srivastava S, Singh Y, Puri P. Pneumopericardium in AIDS. Heart Asia. 2011;3(1):46.

20. Horvat T, Savu C, Motas C, Tetu M. Pneumopericardium - complication of an unknown tuberculosis in a HIV positive patient. Eur J Cardiothorac Surg. 2004;26(5):1043.

21. Weng L, Huang X, Chen L, Feng LQ, Jiang W, Hu XY, Peng JM, et al. Prognostic factors for severe Pneumocystis jiroveci pneumonia of non-HIV patients in intensive care unit: a bicentric retrospective study. BMC Infect Dis. 2016;16(1):528.

22. Bukamur HS, Karem E, Fares S, Al-Ourani M, Al-Astal A. Pneumocystis Jirovecii (carinii) pneumonia causing lung cystic lesions and pneumomediastinum in non-HIV infected patient. Respir Med Case Rep. 2018;25:174-176.

23. Mu XD, Jia P, Gao L, Su L, Zhang C, Wang RG, Wang GF. Relationship between Radiological Stages and Prognoses of Pneumocystis Pneumonia in Non-AIDS Immunocompromised Patients. Chin Med J (Engl). 2016;129(17):2020-2025.

24. Mu XD, Gao L, Wang RG, Su L, Zhang C, Que CL, Li $\mathrm{HC}$, et al. [Retrospective analysis of relationship between radiological features and prognoses of pneumocystis pneumonia in non-AIDS immunocompromised patients]. Zhonghua Yi Xue Za Zhi. 2012;92(38):2703-2706.

25. Clancy DJ, Lane AS, Flynn PW, Seppelt IM. Tension pneumomediastinum: A literal form of chest tightness. J Intensive Care Soc. 2017;18(1):52-56.

26. Lee JC, Bell DC, Guinness RM, Ahmad T. Pneumocystis jiroveci pneumonia and pneumomediastinum in an anti-TNFalpha naive patient with ulcerative colitis. World J Gastroenterol. 2009;15(15):1897-1900.

27. Vidal S, de la Horra C, Martin J, Montes-Cano MA, Rodriguez E, Respaldiza N, Rodriguez F, et al. Pneumocystis jirovecii colonisation in patients with interstitial lung disease. Clin Microbiol Infect. 2006;12(3):231-235.

28. Wang DD, Zheng MQ, Zhang N, An CL. Investigation of Pneumocystis jirovecii colonization in patients with chronic pulmonary diseases in the People's Republic of China. Int J Chron Obstruct Pulmon Dis. 2015;10:20792085.

29. Canas-Arboleda A, Hernandez-Florez C, Garzon J, Parra-Giraldo CM, Burbano JF, Cita-Pardo JE. Colonization by Pneumocystis jirovecii in patients with chronic obstructive pulmonary disease: association with exacerbations and lung function status. Braz J Infect Dis. 2019;23(5):352-357.

30. Esteves F, Lee CH, de Sousa B, Badura R, Seringa M, Fernandes C, Gaspar JF, et al. (1-3)-beta-D-glucan in association with lactate dehydrogenase as biomarkers of Pneumocystis pneumonia (PcP) in HIV-infected patients. Eur J Clin Microbiol Infect Dis. 2014;33(7):1173-1180.

31. Lahmer T, da Costa CP, Held J, Rasch S, Ehmer U, Schmid RM, Huber W. Usefulness of 1,3 Beta-D-Glucan Detection in non-HIV Immunocompromised Mechanical Ventilated Critically Ill Patients with ARDS and Suspected Pneumocystis jirovecii Pneumonia. Mycopathologia. 2017;182(7-8):701-708.

32. Engsbro AL, Najat S, Jorgensen KM, Kurtzhals JAL, Arendrup MC. Diagnostic accuracy of the 1,3-beta-Dglucan test for pneumocystis pneumonia in a tertiary university hospital in Denmark: A retrospective study. Med Mycol. 2019;57(6):710-717.

33. Roux A, Gonzalez F, Roux M, Mehrad M, Menotti J, Zahar JR, Tadros VX, et al. Update on pulmonary Pneumocystis jirovecii infection in non-HIV patients. Med Mal Infect. 2014;44(5):185-198.

34. Ko Y, Jeong BH, Park HY, Koh WJ, Suh GY, Chung MP, Kwon OJ, et al. Outcomes of Pneumocystis pneumonia with respiratory failure in HIV-negative patients. J Crit Care. 2014;29(3):356-361.

35. Festic E, Gajic O, Limper AH, Aksamit TR. Acute respiratory failure due to pneumocystis pneumonia in patients without human immunodeficiency virus infection: outcome and associated features. Chest. 2005;128(2):573579.

36. Monnet X, Vidal-Petiot E, Osman D, Hamzaoui O, Durrbach A, Goujard C, Miceli C, et al. Critical care management and outcome of severe Pneumocystis pneumonia in patients with and without HIV infection. Crit Care. 2008;12(1):R28. 